\title{
9. Jahrestreffen der CE-Alken-Preisträger Celle, 20.-28.11.1991
}

\author{
U. Jonas \\ Urologische Klinik, Medizinische Hochschule Hannover
}

\begin{abstract}
Seit 1976 gilt der CE-Alken-Preis als die erstrebenswerteste urologische Auszeichnung, die jedes Jahr verliehen wird. In den zurückliegenden 16 Jahren wurden insgesamt (einschl. 1992) 23 Wissenschaftler mit dieser Würde bekleidet. $\mathrm{Zu}$ der "Familienfeier" in Celle erschienen knapp 50 Teilnehmer, erfreulich war, daß darunter 9 Preisträger waren. In 16 Vorträgen wurden praktisch alle Aspekte der Urologie behandelt, im folgenden fassen die Autoren (Alken-Preisträger und Gäste) ihre Vorträge zusammen:
\end{abstract}

Johan Denil und Dana A. Ohl (Ann Arbor, Hannover) berichteten über die Möglichkeiten der Elektroejakulation: "The Michigan experience":

Elektrostimulation des inneren männlichen Genitale via einer rektalen Sonde ist eine mögliche Behandlung neurogener Ejakulationsstörungen, wenn eine konservative Therapie versagt oder nicht indiziert ist. An der Universität Michigan wurden von 1986 bis November 1991 insgesamt 198 Männer mit Elektroejakulation behandelt. Hauptindikation in diesem Patientengut war Ejakulationsverlust nach Querschnittlähmung, gefolgt von Anejakulation nach radikaler retroperitonealer Lymphadenektomie. Seltenere Indikationen waren Ejakulationsverlust durch Diabetes mellitus, multiple Sklerose oder Meningomyelozele und idiopathische (psychogene) Anejakulation.

Fast immer konnte eine Samenemission initiiert werden. Ein Ejakulat mit adäquater Qualität für artifizielle Insemination, d.h. mindestens 10 Millionen motilen Spermatozoa mit normaler Morphologie, wurde bei $75 \%$ der Querschnittgelähmten und bei $87 \%$ der Männer nach retroperitonealer Lymphadenektomie gewonnen. Bei den zwei Drittel der Patienten mit Kinderwunsch wurden bis zu 6 Zyklen von kombinierter Elektroejakulation und intrauteriner Insemination durchgeführt. Wurde dadurch keine Schwangerschaft erzielt, dann wurden dem Paar andere Formen der assistierten Reproduktion wie z. B. die intratubare Insemination oder In-vitro-Fertilisierung vorgeschlagen. Es konnten, im Durchschnitt nach 4 Zyklen, 49 Schwangerschaften induziert werden. Daraus wurden bis jetzt 35 gesunde Kinder geboren. Die gesamte Schwangerschaftsrate dieses Projektes beträgt $35 \%$ per Paar. Nebenwirkungen waren minimal, selbst die potentiell gefährliche autonome Dysregulation bei hoher Querschnittlähmung ließ sich mittels Nifedipine gut kontrollieren. Nur zwei ernsthafte Komplikationen traten auf: eine mecha-

Akt. Urol. 24 (1993) 47-57

(C) Georg Thieme Verlag Stuttgart · New York nische Sigmaperforation, die einen passageren Anus praeter erforderlich machte, und ein Kammerflimmern bei einem Diabetiker mit ausgeprägter Cardiomyoneuropathie. Beide Komplikationen hatten einen problemlosen Verlauf.

Limitierend für den Erfolg sind die ausgeprägte Asthenozoospermie der Ejakulate nach Elektroejakulation sowie die funktionellen Abnormalitäten, die sowohl durch die Stimulation als auch durch die chronische Anejakulation bedingt sein können. Individuelle prognostische Aussagen aufgrund der Anamnese und der Qualität der Ejakulate konnten bisher nicht gemacht werden, aber in mehreren Studien zeichnen sich Tendenzen ab. In einer prospektiven klinischen Studie wurden die funktionellen Eigenschaften der "Elektroejakulate“ beschrieben und mit denen von Donorsperma verglichen. Nach Elektroejakulation zeigten die Spermatozoa eine signifikant geringere Penetration in Zervikalmukus, eine schlechtere Überlebung während Inkubation und eine geringere Fertilisationskapazität im Hamster-Oozyten-Penetrationstest als normale Spermien. Diese Funktionsparameter waren zwar höher, aber nicht statistisch signifikant besser bei den Patienten, die später eine Schwangerschaft erreichten. Für die computerassistierte Analyse der Elektroejakulate fanden wir in diesem Vergleich signifikant bessere Parameter.

Diese Studie ist aber noch nicht abgeschlossen, und die bisherigen Fallzahlen sind für eine gute statistische Analyse zu gering. Zusammenfassend sahen wir in den untersuchten Tests bisher Trends zu besseren Ergebnissen bei den Patienten, die später erfolgreich waren, aber noch keine signifikanten Unterschiede. Wir können deshalb noch keinen prognostisch verläßlichen Test beschreiben. Andere Autoren haben in vitro nachgewiesen, daß die elektrische Energie und/oder die Temperaturerhöhung während Elektroejakulation die Qualität der Ejakulate (im routinemäßigen mikroskopischen Untersuchungsverfahren) verringert. Im Hundemodell haben wir den möglichen Einfluß der elektrischen Energie in vivo untersucht. Die mikroskopischen Eigenschaften von Ejakulat - gewonnen in einer artifiziellen Vagina - war jedoch nicht signifikant besser als die der Elektroejakulaten.

Elektroejakulation mit artifizeller Insemination ist eine wirksame und komplikationsarme Behandlung der männlichen Infertilität, bedingt durch neurogene Anejakulation. Laufende und künftige Untersuchungsprojekte richten sich nicht nur auf die Verbesserung der Methode und deren Ergebnisse, sondern auch auf die Identifizierung von besseren prognostischen Tests. So werden wir in der Lage sein, die Patienten, die nicht profitieren werden, früher zu identifizieren. 


\section{Literatur}

1 Denil, J., D. A. Ohl, A. C. Menge, L. Keller, M. McCabe: Functional charateristics of sperm obtained by electroejaculation in men with anejaculator infertility. J. Urol. 147 (1992) 69-72

2 Ohl, D. A., J. Denil, C. J. Bennett, J. F. Randolph, A. C. Menge, M. McCabe: Electroejaculation following retroperitoneal lymphadenectomy. J. Urol. 145 (1991) 980-983

3 Ohl, D., R. Grainger, C. Bennett, J. Randolph, S. Seager, $M$. McCabe: Successful use of electroejaculation in two multiple sclerosis patients including a report of a pregnancy utilizing intrauterine insemination. Neurourology and Urodynamics 8 (1989) 195-198

4 Randolph, J. J., D. A. Ohl, C. J. Bennett, J. W. Ayers, A. C. Menge: Combined electroejaculation and in vitro fertilization in the evaluation and treatment of anejaculatory infertility. J. In Vitro Fert. Embryo Transf. 7 (1990) 58-62

5 Steinberger, R. E., D. A. Ohl, C. J. Bennett, M. McCabe, S. C. Wang: Nifedipine pretreatment for autonomic dysreflexia during electroejaculation. Urology 36 (1990) 228-231

${ }^{6}$ Stewart, D. E., D. A. Ohl: Idiopathic anejaculation treated by electroejaculation. Int. J. Psychiatry Med. 19 (1989) 263-268

C. E. Constantinou (Stanford, USA) präsentierte seine Ergebnisse über den Einfluß der radikalen Prostatektomie auf die Charakteristiken von Blase und Urethra:

In einer prospektiven urodynamischen Studie wurden die Häufigkeit der Detrusorstabilität und die Langzeiteffekte der radikalen Prostatektomie auf die Blasenfunktion untersucht. Die präoperativen Ergebnise bei 21 Männern, die im gemittelten Alter von 63 Jahren radikal prostatektomiert wurden, zeigten, daß bei 16 Patienten Blasendetrusorinstabilitäten mit maximalen Kontraktionsdrucken von $59+/-28 \mathrm{~cm} \mathrm{H}_{2} \mathrm{O}$ resultierten. Etwa 23 Monate postoperativ wurde bei 13 dieser Patienten eine urodynamische Nachuntersuchung durchgeführt. Postoperativ zeigte sich, daß der maximale Detrusordruck bei Instabilität mit $49+/-17 \mathrm{~cm} \mathrm{H}_{2} \mathrm{O}$ nicht signifikant abnahm. Der Vergleich der prä- und postoperativen urodynamischen Charakteristiken bei Blasenfüllung machte deutlich, daß eine radikale Prostatektomie keine signifikanten Veränderungen in den Füllcharakteristiken der Blase zeigte, wenn das Gefühl der vollen Blase oder Harndrang vorhanden waren. Die Druckflußkurven zeigten einen signifikanten Anstieg der maximalen Flußrate von $8+/-1$ auf $13+/-2 \mathrm{ml} / \mathrm{s}(\mathrm{p}$ $=0,007$ ) und eine signifikante Abnahme des maximalen Detrusordruckes, des Urethralöffnungsdrucks und des Restharns. Die Messung des urethralen Druckprofils zeigte keinen signifikanten Unterschied weder in dem maximalen Urethralflußdruck noch in der Länge des externen Sphinkters. Nach Prostatektomie konnte ein Blasenhalsdruck nicht mehr gemessen werden. Dies bewies, daß die Abnahme der obstruktiven Charakteristiken durch die Prostataentfernung bedingt war. Es zeigte sich jedoch, daß Detrusorinstabilitäten bei Prostata-Karzinom-Patienten häufig auftraten, die durch eine radikale Prostatektomie nicht $\mathrm{zu}$ verhindern waren. Bei Patienten mit BPH war die Inzidenz der Detrusorinstabilität häufiger. 3 von den 13 Patienten waren postoperativ inkontinent und bei allen dieser Patienten war der Detrusor prä- und postoperativ instabil. Alle 5 Patienten mit einer präoperativen, urodynamisch bewiesenen Detrusorstabilität waren postoperativ kontinent, trotzdem einige einen geringen urethralen Verschlußdruck und eine kurze funktionelle Harn- röhre aufwiesen. Patienten mit stabilen Blasen erreichten schneller eine postoperative Kontinenz als die Patienten mit Detrusorinstabilität. Urodynamische Studien nach radikalen Prostatektomien mit einem kürzeren postoperativen Verlauf wurden von Presti (2) berichtet. Diese Ergebnisse unterscheiden sich von denen von Hellstrom (1) dadurch, daß er eine viel geringere präoperative Detrusorinstabilität festgestellt hatte. Die Autoren schlossen daraus, daß einige der Patienten mit präoperativen Detrusorinstabilitäten postoperativ inkontinent würden.

\section{Literatur}

${ }^{1}$ Hellstrom, P., O. Lukkarinneno, $M$. Kuntturi: Urodynamics in radical prostatectomy. Scand. J. Urol. Nephrol. 23 (1990) 21 24

2 Presti, J. C., R. A. Schmidt, P. A. Narayah, P. R. Carol, E. A. Tanagho: Pathophysiology of urinary incontinence after radical prostatectomy. J. Urol. 143 (1990) 475-978

Aus der Arbeitsgruppe um H. J. Tanke (Leiden, Niederlande) berichtete $A . K$. Raap über die nicht radioaktive In-situ-Hybridisierung zur klinischen Zytogenetik:

Die Entdeckung (1959) der engen Beziehung zwischen klinischen Syndromen und abnormalen chromosomalen Verhältnissen führte zu einem zunehmenden Interesse an Anzahl und Form von Chromosomen in der menschlichen metaphasischen Zelle. Anfangs war die zytogenetische Analyse sehr schwierig, alle Chromosomen oder chromosomalen Teile individuell ohne Zweifel zu erkennen. Die sogenannte "banding"-Methode der späteren 60er und frühen 70er Jahre half, die Aufdeckungsgenauigkeit zu verbessern und führte letztlich zu immer besseren Informationen über das zytogenetische „Make up“ der (Tumor)zellen. Im selben Zeitraum entwickelte sich die In-situ-Hybridisierung (ISH), mit der es gelang, molekulare DNA-Daten von morphologisch intakten Zellen und Chromosomen zu erhalten, oft "molekulare Zytogenetik“ genannt. Dies gelang insbesondere durch die nichtradioaktive ISH-Methode (2), durch die die molekulare Zytogenetik sowohl experimentell als auch klinisch Anerkennung fand. Die Fähigkeit der ISH-Techniken, Chromosome und /oder Chromosomteile in den Interphasen aufdecken zu können, trug signifikant dazu bei, ISH zu fördern, denn zum ersten Mal hat sich dadurch die Möglichkeit der zytogenetischen Analysen von Zellen ergeben, die nicht oder nur sehr schwer in eine Mitose zu bringen sind, wodurch viel bessere Ergebnisse als bei den Metaphasen Zytogenetiken möglich sind (1).

Die molekulare zytogenetische Information ist in der Anzahl der Hybridisationsdomänen, ihrer Farbe, ihrer spatialen Beziehung und ihrer Intensität enthalten. Abweichungen vom Normalverhalten erlauben Zellen mit einer oder mehreren chromosomalen Aberrationen zu identifizieren. So können z.B. numerische Aberrationen einschließlich von Gendeletionen für verschiedene Chromosome oder Teile daraus einfach durch das Zählen der Anzahl verschieden farbiger Hybridisationsdomänen in der Meta- und Interphase entdeckt werden. Es sind jedoch auch strukturelle Aberrationen wie Translokationen oder Inversionen einfach an der Mischung und/oder Trennung der Farben zu erkennen, die von denen der 
Proben benachbarten Bruchpunkte abstammen. Zusammenfassend läßt sich feststellen, daß ISH-Techniken exzellente Möglichkeiten der Charakterisierung von genetisch abweichenden Zellen bieten, aber auch für die Diagnose und das Aufzeichnen von angeborenen oder erworbenen genetischen Erkrankungen dienlich sind, da krankheitsspezifische Hybridisationsstrategien entwickelt werden können. Es ist zu erwarten, daß in der experimentellen oder auch spezifisch klinischen Zytogenetik die Anzahl der molekularen zytogenetischen Analysen in den kommenden Jahren drastisch ansteigen wird, die zur Entdeckung von neuen und neu zu definierenden Beziehungen zwischen klinischen Syndromen und abnormalen chromosomalen Kostitutionen führen wird.

\section{Literatur}

1 Cremer, T., J. Landegent, A. Bruckner, H. P. Scholl, M. Schardin, H. D. Hager, P. Devilee, P. L. Pearson, M. Van der Ploeg: Detection of chromosome aberrations in the interphase nucleus by visualization of specific target DNAs with radioactive and nonradioactive in situ hybridization: diagnosis of trisomy 18 with L 1,84. Hum. Genet. 74 (1986) 346-352

${ }^{2}$ Raap, A. K., P. M. Nederlof, R. W. Dirks, J. C. A. G. Wiegant, $M$. Van der Ploeg: Use of haptenized nucleic acid probes in fluorescent in situ hybridization. In: In situ hybridization: application to developmental biology and medicine (eds. N. Harris and D. G. Williams, Cambridge University Press). Cambridge, (1990) pp 33-41

K. H. Kurth und Mitarb. (Amsterdam/Niederlande) fassen ihre Ergebnisse über das oberflächliche Blasenkarzinom und insbesondere um seine lokale und zentrale $\mathrm{Pa}$ thologie wie folgt zusammen:

Tumorstadium und Malignitätsgrad sind für die Prognose des Blasenkarzinoms entscheidende Parameter. Dies trifft für die Voraussage der Wahrscheinlichkeit eines Tumorrezidivs und der möglichen Progression ( $\geq$ T2) des oberflächlichen Karzinoms Ta-T1 zu. Therapeutische Überlegungen werden durch Stadium und Malignitätsgrad bestimmt.

In mehreren EORTC-Protokollen zur Behandlung des oberflächlichen Blasenkarzinoms wurde in einer Zusatzuntersuchung die Übereinstimmung der Beurteilung des Malignitätsgrades und der T-Kategorie durch lokale und zentrale Pathologie überprüft. Der zentralen Pathologie (F. t. K.) wurden jeweils zwei Präparate jeglichen Resektionsmaterials (exophytischer Tumor, Biopsie Tumorrand und Tumorgrund, Random-Biopsie) zur Verfügung gestellt. Neben T-Kategorie und Malignitätsgrad wurde eine Reihe zusätzlicher pathologischer Faktoren wie Kernanaplasie, Kernhyperchromasie, Kernkohärenz, Mitose, Epithelverlust und andere bestimmt.

Dem zentralen Pathologen stand Material von 318 Patienten (Prot. 30790) zur Beurteilung zur Verfügung. Tab. 1 stellt die lokale Diagnose der des zentralen Pathologen gegenüber. Die größte Diskrepanz ergab sich bei der Einschätzung der T-Kategorie. Von 133 Patienten mit einem Blasentumor T1 nach der Diagnose des lokalen Pathologen stimmte der zentrale Pathologe lediglich bei $58 \mathrm{~Pa}$ tienten $(44,3 \%)$ mit der Beurteilung überein. Eine größere Übereinstimmung fand sich bei Patienten mit einem Blasentumor der Kategorie Ta. (Übereinstimmung bei 148 /
Tab. 1 T-Kategorie und Malignitätsgrad durch lokale und zentrale Pathologie.

\begin{tabular}{rrrrrrrrr}
\hline & & \multicolumn{7}{c}{ zentral } \\
& & T0 & TIS & \multicolumn{1}{c}{ TA } & T1 & T2 & TX & total \\
\hline & T0 & 0 & 0 & 1 & 0 & 0 & 0 & 1 \\
L & TIS & 2 & 1 & 8 & 0 & 0 & 0 & 11 \\
0 & TA & 1 & 4 & 148 & 21 & 0 & 1 & 175 \\
K & T1 & 2 & 1 & 66 & 58 & 4 & 0 & 131 \\
A & T2 & 0 & 0 & 0 & 0 & 0 & 0 & 0 \\
L & TX & 0 & 0 & 0 & 0 & 0 & 0 & 0 \\
\hline total & & 5 & 6 & 223 & 79 & 4 & 1 & 318 \\
\hline
\end{tabular}

EORTC 30790

\begin{tabular}{|c|c|c|c|c|c|c|c|}
\hline \multicolumn{2}{|c|}{ zentral } & \multirow{2}{*}{$\frac{\text { Go }}{0}$} & \multirow{2}{*}{$\frac{\mathrm{G} 1}{13}$} & \multirow{2}{*}{$\frac{G 2}{17}$} & \multirow{2}{*}{$\frac{\mathrm{G} 3}{2}$} & \multirow{2}{*}{$\frac{G X}{0}$} & \multirow{2}{*}{$\frac{\text { total }}{32}$} \\
\hline L & G0 & & & & & & \\
\hline 0 & $\mathrm{G} 1$ & 3 & 67 & 55 & 9 & 1 & 135 \\
\hline K & G2 & 2 & 10 & 75 & 26 & 0 & 113 \\
\hline A & G3 & 0 & 0 & 6 & 20 & 0 & 26 \\
\hline $\mathrm{L}$ & $G X$ & 0 & 0 & 1 & 1 & 0 & 2 \\
\hline to & & 5 & 90 & 154 & 58 & 1 & 308 \\
\hline
\end{tabular}

EORTC 30790

175 Patienten bzw. bei 84,5\%). Bei der Beurteilung des Malignitätsgrades fand sich Übereinstimmung bei G1-Tumoren in $49,6 \%$, bei G2-Tumoren in $66,4 \%$ und bei G3Tumoren in $76,9 \%$, global stimmten lokale und zentrale Pathologie in $162 / 274$ überein $(59,1 \%)$. Im allgemeinen neigte die lokale Pathologie im Vergleich zum zentralen Pathologen zur Überschätzung der T-Kategorie und zur Unterschätzung des Malignitätsgrades. Andererseits wurde in vier Fällen ein muskelinfiltrierender (T2) Tumor durch die lokale Pathologie nicht erkannt.

Es drängt sich die Frage auf, welche der beiden Pathologien die glaubwürdigere Diagnose stellte. Die Antwort kann aus dem weiteren Verlauf gegeben werden. Alle untersuchten Patienten wurden zwischen 6 Monaten bis 9 Jahren verfolgt, im Mittel 5 Jahre. Werden Rezidivhäufigkeit und Überleben dem Tumorstadium und Malignitätsgrad korreliert, ergibt sich eine bessere diskriminierende Voraussage, wenn beim Vergleich (Ta versus T1, G1 versus $\mathrm{G} 2 / \mathrm{Ge}$ ) Stadiumsbeurteilung und Malignitätsgrad des zentralen Pathologen herangezogen werden (Tab. 2).

Die übrigen pathologischen Faktoren wurden im multivariaten Modell auf ihren Aussagewert einer möglichen Tumorprogression ( $\geq \mathrm{T} 2$ ) untersucht. Hierbei ergab die Kombination Mitose (geringe, mittlere und größere Häufigkeit) und Epithelverlust (gering oder schwergradig) den größten Voraussagewert. Bei geringer Mitosehäufigkeit und geringem Verlust des Epithels betrug die Häufigkeit der Progression $6 \%$, bei großer Häufigkeit von Mitosen und schwergradigem Epithelverlust $46 \%$. 
Tab. 2 Statistische Analyse von rezidivfreiem Interval und Überleben in Abhängigkeit von T-Kategorie und Malignitätsgrad des Blasentumors. Die größere Wahrscheinlichkeit eines Rezidivs und das erhöhte Risiko eines Tumortodes bei Tumoren der Kategorie T1 wird deutlicher, wenn die T-Kategorie durch den zentralen Pathologen festgelegt wurde.

\begin{tabular}{|c|c|c|c|c|c|}
\hline & & & & \multicolumn{2}{|c|}{ p-Wert } \\
\hline & & & & Rezidiv & Überleben \\
\hline $\mathrm{Ta}$ & versus & T1 & (lokal) & 0,05 & 0,04 \\
\hline $\mathrm{Ta}$ & versus & $\mathrm{T} 1$ & (zentral) & 0,0003 & 0,006 \\
\hline G1 & versus & $G 2 / G 3$ & (lokal) & 0,001 & 0,03 \\
\hline G1 & versus & $\mathrm{G} 2 / \mathrm{G} 3$ & (zentral) & 0,001 & 0,004 \\
\hline
\end{tabular}

EORTC 30790

Aus den Ergebnissen können die folgenden Schlußfolgerungen gezogen werden:

- Die lokale Pathologie neigt zur Überschätzung der TKategorie und zur Unterschätzung des Malignitätsgrades.

- Die höchste Übereinstimmung zwischen lokaler und zentraler Pathologie findet sich bei Tumoren der Kategorie $\mathrm{Ta}(84,5 \%)$ und Tumoren mit einem Malignitätsgrad G3 $(76,9 \%)$.

- Mitosen und gestörter Epithelaufbau (Verlust des 5schichtigen Epithelaufbaus) sollten klassifiziert werden. Beide Faktoren besitzen Voraussagewert hinsichtlich der Rezidivhäufigkeit und der Möglichkeit der Tumorprogression.

- Schwerwiegende Entscheidungen (Zystektomie bei T1G3 Tumoren) sollten auch unter dem Aspekt der Irrtumsmöglichkeit der lokalen Pathologie diskutiert werden.

Ähnliche Erfahrungen mit lokaler und zentraler Pathologie berichteten Parmar und Mitarb. Ooms und Mitarb. wiesen auf die inter- und intraindividuellen Abweichungen des Pathologen bei der Beurteilung von Blasentumorpräparaten hin.

\section{Literatur}

1 Ooms, E. C. M., A. P. R. Blok, R. W. Veldhuizen: The reproducibility of a quantitative grading system of bladder tumors. Histopathology 9 (1985) 501-509

2 Parmar, M. K. B., L. S. Freedman, T. B. Hargreave, D. A. Tolley: Prognostic factors for recurrence and followup policies in the treatment of superficial bladder cancer: report from the British medical research council subgroup on superficial bladder cancer (Urological cancer working party). J. Urol. 142 (1989) 284-288
Ebenfalls aus der Arbeitsgruppe um Herrn Kurth (Amsterdam) berichtete D. H. J. Schamhart über die BCGBehandlung beim oberflächlichen Blasenkarzinom, insbesondere über die prognostischen Zytokine und die Verbesserung der Therapie:

Die Immuntherapie mit intravesikalen Instillationen von BCG zur Behandlung und Prophylaxe des Übergangszellkarzinoms der Blase ist heute allgemein anerkannt. Die BCG-Behandlung ist zumindest gleich effektiv oder sogar der allgemein verwendeten Chemotherapie überlegen, aber auf der anderen Seite jedoch wahrscheinlich die am meisten toxische Behandlung beim Blasenkarzinom, bei der auch die höchste Inzidenz von Nebeneffekten feststellbar ist. Bis heute ist das detaillierte Wissen über den Wirkungsmechanismus als eine unspezifische Immunstimulanz und die antitumoröse Modalität unzureichend. Daraus folgt, daß die Grundlagenforschung sich erstens auf die Untersuchung der prognostischen Parameter, den Verlauf und die Therapie und zweitens auf die Verbesserung der Therapie und eine Verminderung der Nebeneffekte richten muß.

Während 14-15 Therapiezyklen wurde der durchschnittliche IL2 und TNF-alpha Titer bestimmt. Es zeigte sich, daß eine BCG-induzierte IL2 Reaktion zum Therapieende auftrat, während die Erhöhung des TNF-alpha Titers bereits während der Therapie manifest wurde (Abb.1a). Diese Beobachtung bestätigt die Fragwürdigkeit, prognostische Schlußfolgerungen zur BCG-induzierten Zytokinrespons zu ziehen. Dagegen scheint das simultane Monitoring von sowohl IL2 als auch TNF-alpha prognostisch wertvoll zu sein (Abb. 1 b).

Die Ursache eines Fehlens klinischer Responz zur „Standard"-BCG-Therapie kann durch eine reduzierte Adhärenz von BCG am Blasenurothel begründet sein. So liegt es auf der Hand, daß es wichtig ist, zu prüfen, inwieweit eine Verbesserung der Adhärenz an der Blasenschleimhaut möglich ist. Pentosan Polysulfat (PPS), ein Molekül, das vergleichbar ist mit den Glycosaminoglycanen der Blasenschleimhaut, das die Blase gegen toxische Produkte schützt und das Anheften von Bakterien verhindert, wurde bereits als Medikament verwendet, um chronische und strahlenbedingte Zystitiden zu behandeln. Zunächst war geplant, damit die BCG-induzierte Zystitis zu beeinflussen. In Tierversuchen wurde begonnen, die BCG-Adhärenz durch eine Vorbehandlung mit PPS (2) zu modulieren. Die Erwartung lag nahe, daß die Gabe von PPS vor einer BCG-Behandlung eine Zystitis verhindern könnte, eben als einen erwünschten Nebeneffekt. Trotzdem ist bis jetzt nichts über eine potentielle Interaktion von PPS mit der Effizienz der BCG-Behandlung bekannt. Dazu sind Tiermodellversuche erforderlich. Die Ergebnisse zeigten nach Gabe von ausreichenden Konzentrationen bei der Ratte, beim Meerschweinchen und bei der menschlichen Blase eine starke Bindung von PPS an der Blasenwand. Darüber hinaus wurde im Gegensatz zu der im allgemeinen durch Bakterien verursachten Zystitis (im Urin) eine signifikante Adhärenz von PPS an Mykobakterien: 1,8, 3,6 und 3,1 $\mu \mathrm{g}$ /mg Trockengewicht von BCG Connaught, RIVM und Pasteur wurden mit $0,2,0,3,0,7$ und $0,0 \mu \mathrm{g} / \mathrm{mg}$ Trockengewicht von E. coli, Streptococus faecalis, Klebsiella pneumoniae und Proteus verglichen. Es wurde das Meerschwein als experimentelles Modell gewählt, das für die BCG-Untersuchungen gut geeignet erscheint. Nach 6wö- 


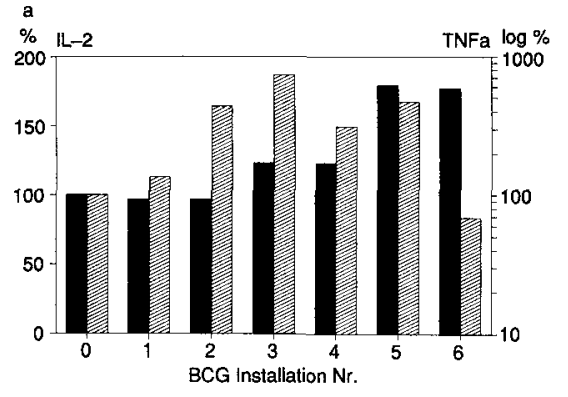

b

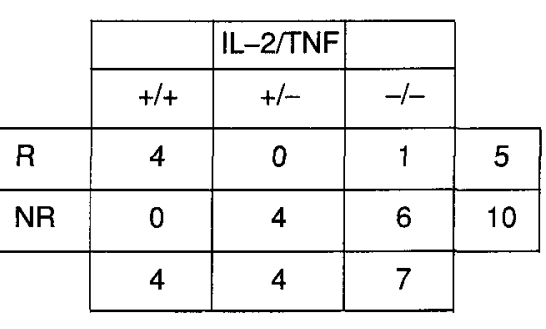

Abb. 1a Maximale Harn IL-2 (schwarze Säulen) und TNF (gestrichelte Säulen) Titer nach BCG-Instillationen bei 14-15 Patienten, ausgedrückt als Prozentsatz der PrätherapieTiter.

1b Anzahl der klinischen Responder $(R)$ und nicht-Responder (NR) mit einem erhöhten IL2 und TNF $\alpha(+/+)$, Erhöhung sowohl des IL-2 oder TNF $\alpha(+/-,-/+)$ oder ohne Erhöhung $(-/-)$. Die Entscheidungskriterien waren: „Tumorfreiheit" für mehr (R) oder weniger (NR) als 12 Monate und "positiv", wenn die maximale Zytokinkonzentration nach Instillation 46 die Prätherapie durchschnittlich um 1SD (IL-

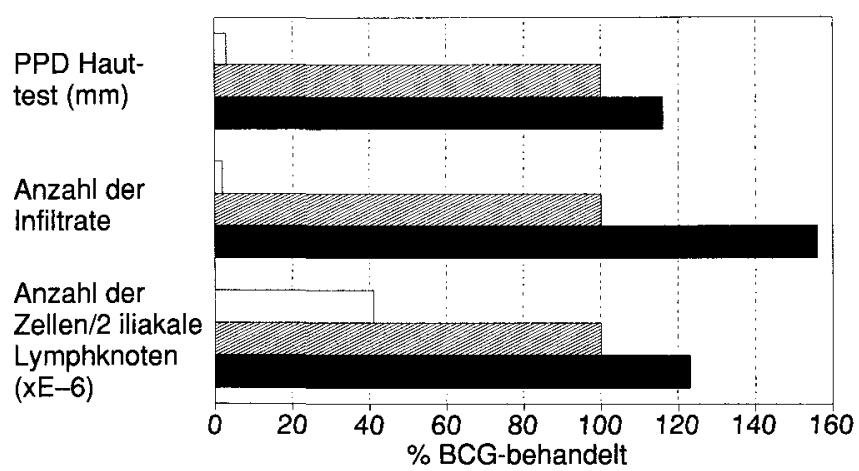

Placebo Fen BCG

$P P S+B C G$

Abb. 2 Effekt der PPS-Vorbehandlung auf verschiedene Parameter in Beziehung zur BCG-assoziierten Immunrespons beim Meerschwein. Die Respons der Parameter wurde relativ zu denen ausgedrückt, die nach der 6. BCG-Instillation gefunden wurden.

chentlichen Instillationen von BCG-RIVM $\left(1 \times 10^{7}\right.$ CFU $)$ mit oder ohne vorheriger Instillation mit PPS $(10 \mathrm{mg}$ in 1 $\mathrm{ml}$ für $1 / 2$ Stunde) resultierten in der 6 . Woche in einer erhöhten PPD Hautreaktion, mononuklearen Infiltrationen in der Blasenwand und einer erhöhten Anzahl von Zellen in den Lymphknoten (Abb. 2).

PPS scheint im Meerschweinchenmodell neben einer Entzündungsreaktion auch eine BCG-induzierte Immunrespons zu stimulieren, auch wenn dies bis heute noch nicht signifikant bewiesen ist. Diese Resultate scheinen zu bestätigen, daß PPS die Adhärenz von BCG an der Blasenwand erhöht, dies kann zu einer Erhöhung der Effektivität der Therapie führen.

\section{Literatur}

1 Schamhart, D. H. J., K. H. Kurth, Th. M. de Reijke, R. Vleeming: BCG treatment and the importance of an inflammatory response. Urol. Res. 20 (1992) in press

2 Schamhart, D. H. J., L. C. de Boer, R. F. M. Bevers, K.H. Kurth, $P$. A. Steerenberg: Mycobacterial adherence and BCG treatment of superficial bladder cancer. Prog. Clin. Biol. Res. (1992) in press
B. J. Schmitz-Dräger und Mitarb. aus Düsseldorf berichten über monoklonale Antikörper gegen normales Urothel und Urotheltumoren - Isolierung von Isotyp-Mutanten

Hauptproblem des In-vivo-Einsatzes von monoklonalen Antikörpern (mAk) ist die geringe Anreicherung von $\mathrm{mAk}$ im Tumor, die von der Pharmakokinetik der mAk abhängig ist. Die Pharmakokinetik der Antikörper wird ganz entscheidend durch den Subtyp determiniert. Da mAk identischer Spezifität jedoch verschiedenen Isotyps bisher nicht vorhanden waren, konnten systematische, vergleichende Untersuchungen zur Pharmakokinetik und biologischen Aktivität bisher nicht durchgeführt werden. Ziel der vorliegenden Arbeit war es, mAk identischer Spezifität mit verschiedenen Isotypen zu identifizieren und isolieren. Ausgangspunkt waren die mAk Due ABC 1, Due ABC 3, Due ABC 5 und Due AUT 2, die gegen normales Urothel und/oder Urotheltumoren gerichtet sind. Durch den Einsatz isotypspezifischer Nachweisantikörper in einem selbst entwickelten Enzyme linked Immuno-sorbent Assay (ELISA) gelang der Nachweis von Isotypmutanten, deren Isotyp von IgM nach IgG 1 (Due $A B C$, Due $A B C 3$, Due $A B C$ 5) bzw. von IgG 1 nach $\operatorname{IgG}_{2 a}$ und IgG $_{2 b}$ (Due AUT 2) gewechselt war. Für die mAk Due ABC 1 und Due AUT 2 wurden die Isotypmutanten mit Hilfe des Sequential Sublining (SSL) kloniert. Durch immunhistochemische Untersuchungen sowie durch Kompetitionstests an permanenten Zellinien von Harnblasenkarzinomen konnte die unveränderte Spezifität der Isotypmutanten nachgewiesen werden. Die Avidität der mutanten Due ABC1 IgG 1 Antikörper war gegenüber dem ursprünglichen IgM erniedrigt. Die mutanten Due AUT $2 \operatorname{IgG}_{2 a}$ und $\operatorname{IgG}_{2 b}$ hatten hingegen die gleiche Avidität wie die ursprünglichen $\operatorname{IgG}_{1}$ Antikörper. Untersuchungen der komplementabhängigen Zytotoxizität (CDC) zeigten, daß nur mAk Due AUT 2 des mutierten Isotyps $\operatorname{IgG}_{2 \mathrm{a}}$, nicht jedoch des ursprünglichen Isotyps $\operatorname{IgG}_{1}$, in der Lage sind, in vitro eine CDC auszulösen. Die Ergebnisse zeigen, daß es mit dem SSL möglich ist, Isotypmutanten mit Spezifität zu generieren, die für pharmakokinetische In-vivo-Untersuchungen eingesetzt werden können. 
R. J. A. van Moorselaar und Mitarb. (Nijmegen, Niederlande), die Alken-Preisträger des Jahres 1990, präsentierten ihre Ergebnisse zur Zytokintherapie beim experimentellen Prostatakarzinom:

Das Prostatakarzinom ist der häufigste Tumor beim Mann, der hauptsächlich ab dem 50. Lebensjahr mit einer zunehmenden Inzidenz auftritt. Somit werden aufgrund der Alterszunahme das Auftreten und die Mortalität auch dieser Erkrankung steigen. Die Hormontherapie ist die Therapie der Wahl bei Patienten mit fortgeschrittenem und metastasierendem Prostatakarzinom. Etwa 60-70\% dieser Patienten sind - zumindest initiell - mit der Androgenablation beeinflußbar, aber praktisch alle diese Patienten zeigen im späteren Verlauf irgendwann einen Relaps, der bis zu einer fehlenden Respons für eine weitere antiandrogene Therapie führt. Eine Therapieoption beim Relaps und beim hormonresistenten metastasierenden Prostatakarzinom könnte die Chemotherapie sein, es ist bis jetzt jedoch noch keine klare Aussage zu führen, inwieweit die Chemotherapie das Leben im Vergleich zur palliativen Therapie verlängert. So haben randomisierte Studien, die die Einzel- und Kombinationsbehandlung der Chemotherapie miteinander verglichen haben, bis heute noch keinen Überlebensvorteil zeigen können. Daher sind neue Untersuchungen erforderlich, um den Wert einer androgenunabhängigen Prostatakarzinomtherapie zu beweisen.

Die Immuntherapie mit den biologischen Response-Modifiers, wie z.B. den Interferonen (IFNs) und dem Tumor Nekrose Faktor Alpha (TNF), wurde bei verschiedenen Formen maligner Erkrankungen erfolgreich angewandt. IFN-gamma ist ein Produkt von aktivierten T-Lymphozyten mit potenten antiviralen, antiproliferativen und immunmodulierenden Eigenschaften, wie z.B. der Aktivierung der natürlichen Killerzellen und Makrophagen, mit einem Anstieg der Expression von tumorassozierten Antigenen und der Modulation von MHC Klasse I und Klasse II Antigen. TNF ist das Zytokin im Mäuseserum, das mit BCG und Endotoxin behandelt wurde und zur Tumorlyse führt. Es ist in vitro in einigen Tumorlinien zytotoxisch bzw. zytostatisch und kann eine hämorrhagische Nekrose im transplantierten Tumor in vivo hervorrufen. TNF wird durch aktivierte Makrophagen produziert.

Wir haben den Antitumoreffekt von IFN und TNF in zwei Tiermodellen untersucht. Die Heterotransplantation von menschlichem Tumor in die nackte Maus bieten ein Modell, mit dem man die Antitumoraktivität von neuen Medikamenten beim menschlichen Tumor in vivo überprüfen kann. Aufgrund des kongenitalen Fehlens vom Thymus sind die zellübertragenden Immunfunktionen bei der nackten Maus stark beeinflußt. Daher fehlt diesen Tieren eine der wichtigsten Effektorzellen, die (zytotoxische) TZelle, und daher kann der Direkteffekt von Zytokinen untersucht werden. Eine Möglichkeit, das komplette Spektrum der zytokinübertragenden Effekte, z. B. der direkten und indirekten hostübertragenden Effekte, untersuchen zu können, könnte die Verwendung von Tumoren in syngenetischen Tieren sein. Wir haben daher auch den antitumoralen Effekt von IFN und TNF gegen Dunning-Rattenprostatatumoren untersucht.

Es wurden zwei etablierte menschliche Prostatakarzinomzell-Linien PC3 und DU145 an der nackten Maus untersucht (1). Subkutan wurde peritumoral alpha- und gam-
ma-IFN und TNF gegeben, beginnend 24 Stunden nach subkutaner Implantation von $1-2 \mathrm{~mm}^{3}$ Tumorstücken. Das IFN-alpha wurde $3 \times$ pro Woche und TNF $5 \times$ pro Woche gegeben. IFN-alpha (Dosis: $0,5-5 \mathrm{ng} / \mathrm{g} \mathrm{Kg}$ ) zeigte einen signifikanten wachstumshemmenden Effekt gegenüber PC3-Tumoren, zeigte jedoch keinen signifikanten Antitumoreffekt gegen den DU145-Tumor. Die IFN-gamma Monotherapie (Dosis: $8-80 \mathrm{ng} / \mathrm{g} \mathrm{Kg}$ ) war weniger effektiv als IFN-alpha. $500 \mathrm{ng} / \mathrm{g}$ TNF produzierte einen Wachstumsstop bei beiden Tumoren, während niedrige Dosen (50 ng/g) nur gegenüber PC3-Tumoren effektiv waren. Die Kombination von IFN-alpha und -gamma hatte signifikante antiproliferative Effekte gegenüber dem PC3-Tumor, jedoch nicht gegenüber dem DU145-Tumor. Kombination von IFN-alpha und TNF waren gegen beide Tumoren effektiv, einige Kombinationen resultierten in einem kompletten Wachstumsstop. IGN-gamma und TNF-Kombinationen zeigten auch einen signifikanten antitumoralen Effekt gegen beide Tumorlinien.

Aufgrund der Spezies-Spezifität von IFN wurde Rattengamma-IFN in dem Rattenprostatatumormodell gewählt (2). Im Gegensatz zu IFN zeigte TNF eine geringere Spezies-Spezifität. In-vitro-Studien am double-layer soft-agarAssay zeigten eine äußerst limitierte anti-proliferative Aktivität der Medikamente in einer Dosis von 1-1000 U IFNgamma und/oder 1-1000ng TNF pro Schale. Für die In-vivo-Studien wurden die Tumoren subkutan implantiert und die Medikamente peritumoral gegeben. Die Behandlung startete 2 Tage nach der Tumorimplantation, IFN-gamma wurde $3 \times$ pro Woche 8000 oder $80000 \mathrm{U}$ pro Ratte und TNF $5 \times$ pro Woche 10 oder $100 \mathrm{ng}$ pro Ratte gegeben. Die IFN-gamma und TNF-Monotherapie waren nicht signifikant effektiv in der Wachstumshemmung androgenabhängiger oder -unabhängiger Tumoren mit der Ausnahme für IFN-gamma gegen den androgenunabhängigen MatLyLu-Tumor. Die Kombination von IFN-gamma und TNF hatten synerge antiproliferative Effekte gegen alle 4 getesteten Tumorlinien, ein kompletter Wachstumsstop konnte jedoch nicht erreicht werden. Überlebensstudien zeigten einen signifikanten Anstieg im Überleben der tumortragenden Ratten.

Die Daten zeigten, da 3 Zytokine einen antiproliferativen Effekt gegen androgenabhängige und -unabhängige Prostatatumoren haben können, dies scheint einen neuen Weg in der Behandlung des Prostatakarzinoms zu ermöglichen.

\section{Literatur}

1 Van Moorselaar, R. J. A., P. van Stratum, G. Borm, F. M. J. Debruyne, J. A. Schalken: Differential antiproliferative activities of alpha- and gamma-Interferon and Tumor Necrosis Factor alone or in combinations against two prostatic cancer xenografts transplanted in nude mice.

2 Van Moorselaar, R. J. A., B. Th. Hendriks, P. van Stratum, P. H. van der Meide, F. M. J. Debruyne, J. A. Schalken: Synergistic antitumor effects of rat-gamma-Interferon and human Tumor Necrosis Factor alpha against androgendependent and -independent rat prostatic tumors. Cancer Res. 51 (1991) $2329-2334$ 
E. P. Allhoff (Hannover) berichtete über den zeitgemäBen diagnostischen Ansatz für die Früherkennung des Prostatakarzinoms mit einer exakten statistischen Aufarbeitung der verschiedenen Früherkennungsmaßnahmen, eine Arbeit, für die der Arbeitsgruppe der Jahrespreis der Niedersächsischen Krebsgesellschaft 1991 zuerkannt wurde:

Seit Isolierung und Charakterisierung des prostataspezifischen Antigens (PSA) durch Wang et al. 1979 hat die Bestimmung des Serumwertes dieses Glykoproteins entscheidende Bedeutung für die Diagnostik und Verlaufskontrolle des Prostatakarzinoms erlangt (1). Während des gleichen Zeitraumes gewann die transrektale Ultraschall-Untersuchung (TRUS) gleichermaßen an Akzeptanz. Inwieweit diese neu hinzugekommenen Modalitäten die Standard-Methode zur Krebsfrüherkennung, die digitale rektale Untersuchung (DRE), ersetzen oder ergänzen können, war Gegenstand jüngster prospektiver Studien. Catalona et al. ermittelten den Stellenwert der PSA-Serumwert-Bestimmung als "first-line“ diagnostische Maßnahme in einer Untersuchung von 1653 Patienten mit einem Lebensalter von mehr als 50 Jahren (2). Bei einem PSA-Wert im Normbereich $(<4 \mathrm{ng} / \mathrm{ml})$ unterblieben weitere Maßnahmen, wogegen Patienten mit einem PSA-Wert $>4 \mathrm{ng} / \mathrm{ml}$ der DRE sowie der TRUS zugeführt wurden. Im Falle eines zweifelhaften Tast- und/oder Ultraschallbefundes erfolgte eine Biopsie wie ebenso bei PSA-Werten $>10 \mathrm{ng} / \mathrm{ml}$. Bei 19/85 Patienten (22\%) mit PSA-Werten zwischen $4-9 \mathrm{ng} / \mathrm{ml}$ und bei $18 / 27$ Patienten (67\%) mit PSA-Werten $>10 \mathrm{ng} / \mathrm{ml}$ bestätigte die feingewebliche Untersuchung die Tumorerkrankung. $32 \%$ der Karzinom-Patienten wäre mit DRE und $43 \%$ mit TRUS allein nicht erfaßt worden. Die Autoren folgern, daß PSA als Ergänzung zur DRE die niedrigste Fehlerwahrscheinlichkeit einer Zwei-Parameter-Kombination aufweist.

Die an der eigenen Institution prospektiv bei bisher über 2500 Patienten erhobenen Befunde von DRE, PSA und TRUS bestätigen - sowohl einzeln als auch in Kombination gewertet - eindrucksvoll die oben genannten Ergebnisse. Nach statistischer Analyse unter ausschließlicher Wertung der histologisch gesicherten Diagnosen erbringt die Kombination der Standard-Methode DRE mit der PSABestimmung in einer "und“-Verknüpfung (d.h. beide Befunde indikativ für $\mathrm{Ca}$ ) eine Verbesserung der Spezifität um fast $20 \%$. Ist in diesem Testansatz nur eine der beiden Untersuchungen suspekt auf ein $\mathrm{Ca}$, so wäre durch nachfolgende Kontrolluntersuchungen als zweitem Raster die Erfassung fast aller Ca-Fälle (97\%) möglich. Eine Dreifachkombination der Einzelverfahren erbrachte ohne inakzeptable Einbußen hinsichtlich Sensitivität und Spezifität kein Informationsplus, so daß sich der Einsatz der TRUS eher für das Staging und Monitoring der Tumorerkrankung empfiehlt.

\section{Literatur}

${ }^{1}$ Allhoff, E. P., W. de Riese, M. Eifinger, J. Pethke, U. Jonas: Prostate-specific antigen - comparative clinical appreciation of a serodiagnostic measure after 8 years of experience. W. J. Urol. 7 (1989) 12-16

${ }^{2}$ Catalona, W. J., D. S. Smith, T. L. Ratliff, K. M. Dodds, D. E. Coplen, J. J. J. Yuan, J. A. Petros, G. L. Andriole: Measurement of prostate specific antigen in serum as a screening test for prostate cancer. New Engl. J. Med. 324 (1991) 1156-1161
Aus der Arbeitsgruppe von Mainz faßte $S$. C. Müller die Ergebnisse zur Möglichkeit der Anwendung des Appendix als Kontinenzmechanismus in der Harnableitung mittels „Mainz Pouch“ zusammen:

Der in der Technik nach Lich-Gregoir in die Taenia libera des Zökums eingebettete Blinddarm (Abb. 3) bietet neben einem idealen Kontinenzmechanismus noch folgende Vorteile:

Das für die Pouchbildung auszuschaltende Darmsegment kann verkürzt werden, da der Invaginationsnippel wegfällt. Kein Nippelgleiten, kein Nippelprolaps mehr.

Kein Fremdmaterial (Metall-Staples) und damit kein Risiko der Pouchsteinbildung.

Verkürzte Operationsdauer.

Problemlos intermittierender Katheterismus.

An insgesamt 28 Patienten untersuchten wir den urodynamischen Aspekt des Blinddarms als Kontinenzmechanismus. Die Untersuchungen wurden intraoperativ vor und nach Einbettung sowie 3 bis 33 Monate postoperativ durchgeführt.

Wir konnten nachweisen, da $\beta$ die anatomische Konfiguration der muskulären Appendixwand keine eigene Sphinkteraktivität besitzt. Der durchschnittliche Maximaldruck eines Appendix-Ruheprofils lag bei $52 \mathrm{~cm} \mathrm{H}_{2} \mathrm{O}(24-204$ $\mathrm{cm} \mathrm{H}_{2} \mathrm{O}$ ), wobei die Druckspitzen in $68 \%$ am Apex der Appendix lagen. Nur $40 \%$ aller gemessenen Appendizes zeigten einen Maximaldruck, der $60 \mathrm{~cm} \mathrm{H}_{2} \mathrm{O}$ überstieg. Daraus kann gefolgert werden, da $\beta$ ein Kontinenzmechanismus durch den Blinddarm allein nicht garantiert werden kann, wenn ein uringefüllter Pouch ungehemmte Kontraktionen aufweist.

Nach der Einbettung der Appendix in die Taenia libera des Zökums im Sinne der Lich-Gregoir Technik kommt es zu einer passiven Drucktransmission der Pouchdrucke auf die Appendix. Zusätzlich konnten wir eine aktive Drucksteigerung nachweisen, die möglicherweise durch eine Kompression der eingebetteten Appendix durch die sich kontrahierende Kolonmuskulatur hervorgerufen wird.

Das Ruhedruckprofil des eingebetteten Blinddarms zeigte eine funktionelle Länge von $25 \mathrm{~mm}(12-60 \mathrm{~mm})$ und einen durchschnittlichen Verschlußdruck von $45 \mathrm{~cm} \mathrm{H} \mathrm{H}_{2} \mathrm{O}$ (16- $96 \mathrm{~cm} \mathrm{H}_{2} \mathrm{O}$ ). Die urodynamischen Ergebnisse aller 28 untersuchten Patienten waren reproduzierbar. Alle Patienten sind komplett kontinent und entleeren den Pouch durch intermittierenden Selbstkatheterismus.

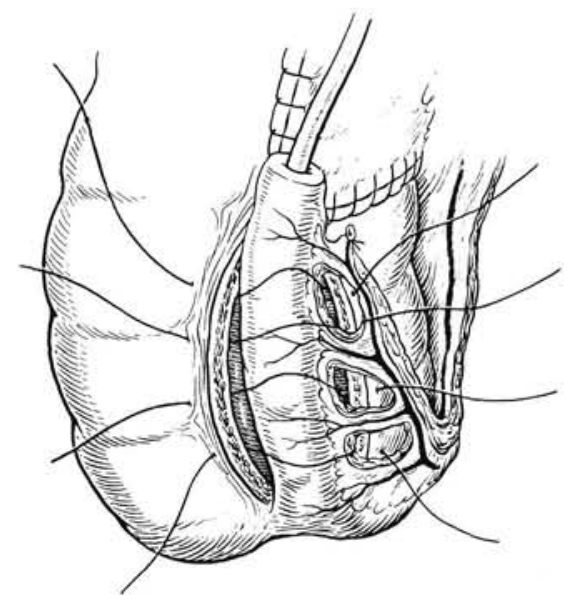

Abb. 3 Submuköse Einbettung der Appendix als kontinentes, katheterisierbares Stoma beim MainzPouch: die Mesoappendix verbleibt außerhalb des submukösen Tunnels. Zum Verschluß der seromuskulären Nähte über der Appendix wird die Mesoappendix unter Schonung des Gefäßverlaufs gefenstert. 


\section{Literatur}

1 H. Riedmiller, R. Bürger, S. C. Müller, J. Thüroff, R. Hohenfellner: A modification of the Mainz Pouch Technique. J. Urol. 143 (1990) $1115-1117$

Zum "State of the art"-Vortrag war Prof. R. Pichlmayr von der Abteilung für Transplantations-Chirurgie der Medizinischen Hochschule Hannover eingeladen worden, um über seine Erfahrungen der Lebertransplantation zu berichten, die in den letzten Jahren spektakuläre Fortschritte gezeigt hat.

Die Lebertransplantation hat sich in den letzten zwei Jahrzehnten zu einer klinischen Behandlungsmethode entwickelt. Am deutlichsten sind die Fortschritte an der Verbesserung der 1-Jahres-Überlebenshöhe abzulesen: Sie lag zu Anfang dieser Entwicklung bei etwa 30-40\% und kann jetzt 80,90 oder $95 \%$ erreichen. Dabei ist das Ergebnis heute vor allem von dem präoperativen Zustand des Patienten abhängig. Die immunsuppressiven Möglichkeiten, wie sie besonders seit Einführung von Cyclosporin A und den poly- und monoklonalen Antikörpern gegeben sind, erlauben es in der Regel, die Abstoßungsreaktion zu verhüten bzw. zu überwinden.

Der Zeitpunkt der Lebertransplantation ist somit eines der wesentlichen Probleme: Er soll so gewählt werden, daß stärkere Allgemeinkomplikationen der Zirrhose noch nicht vorliegen. Leider gelingt es bei der begrenzten Zahl von Spenderorganen nicht immer, die rechtzeitig gestellte Indikation auch tatsächlich zu realisieren.

Die Lebertransplantation bei Malignomen der Leber ist eng begrenzt und hat wohl nur bei wenigen Tumoren, vor allem bei solitären hepatozellulären Karzinomen, bei denen die fortgeschrittene Leberzirrhose eine Leberresektion nicht erlaubt, Berechtigung.

Fortschritte sind in den letzten Jahren besonders auch in der Technik erreicht worden: So können Kinder mit Teilen einer Leber eines Erwachsenen behandelt werden, oder auch eine Leber eines Erwachsenen kann für zwei Patienten entsprechend geteilt und transplantiert werden.

Die wichtigsten Aufgaben für die nächste Zukunft sind wohl der Versuch, möglichst vielen Patienten die Chance einer Lebertransplantation durch Vermehrung der Spenderorganzahlen zukommen zu lassen; weiter sollte die Langzeit-Immunsuppression noch verbessert werden können, um einerseits toxische Nebenwirkungen (z.B. Nephrotoxizität) zu vermeiden und andererseits chronische Abstoßvorgänge soweit wie möglich einzuschränken.

Als Ausdruck der intensiven interdisziplinären Zusammenarbeit zwischen den Kliniken für Urologie und Chirurgie präsentierten E. P. Allhoff und Mitarb. aus beiden Zentren über die chirurgische Therapie von Metastasen beim disseminierten Nierenzellkarzinom:

Die Behandlungsergebnisse beim metastasierten Nierenzellkarzinom sind weiterhin unbefriedigend. Mittels Chemotherapie gelingt es lediglich, in 5-10\% eine Response zu erzielen; die Radiotherapie erlaubt nur eine symptomatische Palliation schmerzhafter Knochenmetastasen. Immuntherapeutische Ansätze zeigen je nach gewähltem $\mathrm{Zy}$ - tokin Responsraten zwischen 10 und $15 \%$ in der Monobehandlung und ca. $30 \%$ bei Kombinationen (1).

In einer retrospektiven Studie von bisher insges. $118 \mathrm{~Pa}-$ tienten mit einem metastasierten Nierenzellkarzinom wurden die Krankheitsverläufe von 20 Patienten analysiert, deren Tumorabsiedlungen unterschiedlicher Lokalisationen (insges. 12) nach Tumornephrektomie chirurgisch therapiert worden waren. Das metastatische Geschehen war in 7 Fällen unifokal, in den übrigen 13 Fällen multilokulär. Die Leber war bei 12 Patienten involviert und damit die am häufigsten betroffene Lokalisation. Die Überlebenszeiten dieser Patienten wurden einer altersentsprechenden Gruppe $(\mathrm{n}=30)$ aus dem Gesamtkollektiv gegenübergestellt. Bei beiden Gruppen konnte ein synchroner bzw. während des Krankheitsverlaufs aufgetretener metachroner Zweittumor ausgeschlossen werden. In der Berechnung nach Kaplan-Meyer wurde für die Patienten mit Metastasenresektion eine 5-Jahres-Überlebensrate von $35 \%$ ermittelt, gegenüber $6,7 \%$ der nicht chirurgisch therapierten Patienten mit disseminierter Tumorerkrankung. Die erhobenen Daten entsprechen der an der Thorax-Klinik Heidelberg ermittelten 5-Jahres-Überlebensrate von $38 \%$ nach chirurgischer Intervention bei Patienten mit einem generalisierten Nierenzellkarzinom (2).

U.E. definieren die erzielten Ergebnisse die Patienten mit unifokaler bzw. multilokulärer Metastasierung beim Nierenzellkarzinom als Untergruppe, bei der eine chirurgische Behandlung der Ansiedlung sinnvoll erscheint. Inwieweit durch Kombination mit einer Immunmodulation darüber hinaus ein signifikanter Überlebensvorteil erreicht werden kann, bleibt Gegenstand weiterer prospektiver Untersuchungen.

\section{Literatur}

1 Allhoff, E. P., S. Liedke, H. Kirchner, J. Atzpodien, W. de Riese, C. G. Stief, $U$. Jonas: - Current clinical relevance of immunotherapy in metastatic renal cell cancer. W. J. Urol. 9 (4) (1991) 228-231

2 Branscheid, D., S. Krysa, G. Wollkopf, H. Bülzebruck, G. Probst, M. Horn, J. Schirren, I. Vogt-Moykopf: - Does ND-Yaglaser extend indication for resection on pulmonary metastases? Eur. J. Cardio-thorac Surg. in press

1991 wurden zwei Arbeitsgruppen mit dem C. E. AlkenPreis ausgezeichnet: G. M. Lennon et al. aus Dublin erhielten diese Auszeichnung für ihre Arbeit mit dem Titel: In vivo versus in vitro Physiologie: Eine Vergleichsstudie am Harnleiter des Kaninchens:

Ein brauchbares und repräsentatives In-vitro-Modell zur Funktionsuntersuchung des Harnleiters könnte eine gute Möglichkeit zum Studium der normalen und pathologischen Harnleiterfunktion unter kontrollierten Laborkonditionen abgeben. In der vorliegenden Studie wurden zwei Modelle für die Messung der Harnleitermotilität am Kaninchen beschrieben. Die in vivo transkortikale Methode bestand aus einer chronisch applizierten Nephrostomiesonde, mit der reproduzierbar Messungen zur Harnleitermotilität durchgeführt werden konnten. Die Kathetergröße, der Typ und die Insertionsroute beeinflußten signifikant die normale ureterale Motilität. Im intakten Harnleiter konnten keine regionalen Variationen der Kontraktili- 
Versuchsmodell:

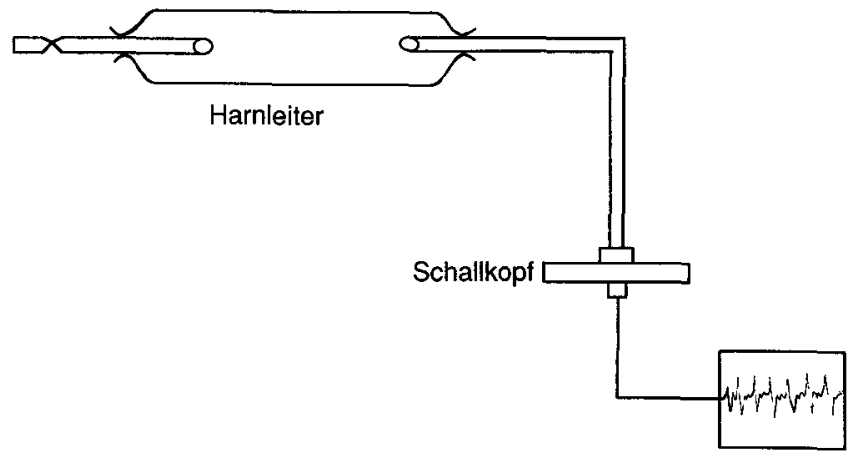

Versuchsanordnung

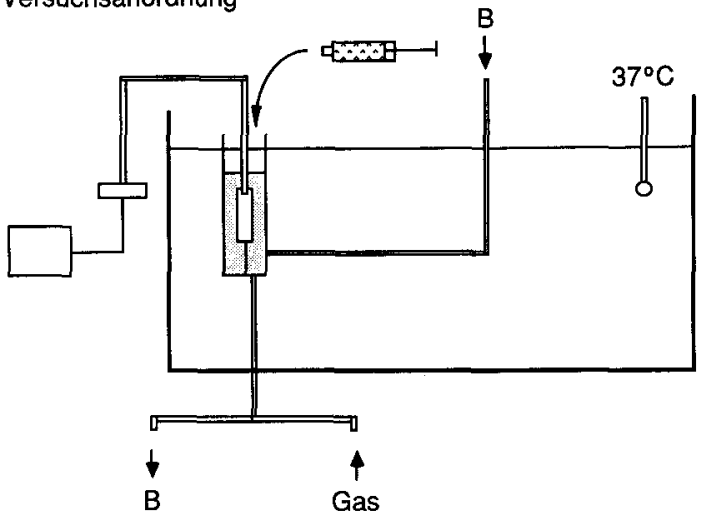

Abb. 4, 5 In-vitro-Modell zur funktionellen Untersuchung der Harnleitermotilität. Erläuterungen s. Text.

tät gefunden werden, es zeigte sich jedoch, daß der obere, mittlere und untere Harnleiter signifikante Unterschiede in der intrinsischen Aktivität zeigten, wenn sich der Harnleiter nicht mehr unter dem steuernden Einfluß der pelvio-calicealen Schrittmacher befand.

Die in vitro segmentalen Uretermodelle (Abb. 4,5) wurden an 72 isolierten Segmenten des Kaninchenharnleiters untersucht. Die Segmente zeigten spontane Kontraktilitäten für Perioden bis zu 12 Stunden, die einen intraluminaren Druck von nur 2-5 mmHg und periodische Druckschübe zur Unterhaltung der spontanen Aktivität brauchten. Diese Aktivität war gegenüber Veränderungen des $\mathrm{pH}$ und der Temperatur resistent. Die regionale Analyse von spontanen Ureteraktivitäten machte deutlich, daß die oberen Segmente gegenüber den Segmenten vom mittleren und distalen Harnleiter eine gleichbleibend kürzere Verzögerung bei Aktivitätsbeginn aufwiesen. Es wurden jedoch keine Unterschiede in der Kontraktilität per se festgestellt, wenn die Aktivität einmal begonnen hatte.

Die Erhöhung des intraureteralen Druckes produzierte einen signifikanten Anstieg sowohl der Rate als auch der Amplitude der Kontraktionen. Dieser Effekt war identisch zu dem, der bei einer akuten mechanischen Okklusion eines intakten Ureters in vivo produziert wurde. Longitudinale Kräfte von 1-50g produzierten einen Anstieg des intraureteren Ruhedrucks, ohne daß dabei ein Wechsel der Rate oder Amplitude der Kontraktion auftrat. Dieser Effekt konnte durch Anwendung von gestaffeltem Zug auf den intakten Ureter reproduziert werden. Eine bakterielle Harntraktinfektion zeigte einen gegenseitigen Effekt bei In-vitro-Kontraktilitätsmessungen, wenn sie selektiv im Ureterlumen oder im Muskel appliziert war.

Vergleichbare Analysen der Harnleitersegmente nach 4 Wochen kompletter und partieller Obstruktion und anschließender Re-Implantation und Erholung zeigte eine verringerte/angestiegene Kontraktilität der ersten bzw. zweiten Gruppe. Die Motilitätsänderungen waren sehr ähnlich sowohl in der in vivo als auch in der In-vitro-Anordnung. Im Fall eines Harnleiters, der chronisch mit einem Doppel-J-Katheter geschient ist, zeigte das In-vitroModell deutlich, daß - wenn die Motilität mechanisch gestört ist - dann In-vivo die potentielle Kontraktilität des Muskels an sich unangetastet bleibt oder sogar erhöht ist.
Diese In-Vitro-Untersuchung des segmentalen Ureters ist eine einfache, verläßliche und quantitative Methode zur in-vitro Beurteilung der ureteralen Kontraktilität. Die wichtigste Voraussetzung von jedem physiologischen Invitro-Modell ist, daß es exakt repräsentativ eine intakte In-vivo-Funktion darstellt. Dieses Kriterium hat sich im isolierten Segment des In-vitro-Ureters klar dargestellt.

G. H. Mickisch erhielt den Alken-Preis für seine Arbeit: Gentransfer, Gentherapie und molekulare Therapiesteuerung - Die Zukunft der Uro-Onkologie

Der klinisch tätige Urologe wird in zunehmendem Maße gefordert, moderne wissenschaftliche Methoden zur Lösung klinischer Probleme einzusetzen. Am Beispiel der Vielfach-Chemoresistenz menschlicher Tumoren kann exemplarisch der Nutzen von Gentechnologie in der klinischen Medizin nachgewiesen werden. Bereits die Klärung einer der wichtigsten Resistenzmechanismen (Abb. 6), die nachfolgende Klonierung und Sequenzierung der verursachenden Gene, und schließlich ihr Nachweis im Tumorgewebe (Abb. 7), brachte Impulse für Diagnostik und experimentelle Therapie u.a. des Nierenzellkarzinoms. Durch Transfer des menschlichen Resistenzgenes MDR1 in Mäuse entstanden immunologisch intakte Tiere, die im exprimierenden Organ, z. B. im Knochenmark, funktionell chemoresistent sind. Damit bieten sich neue Möglichkeiten, die Funktion des Resistenzgenes, z. B. unter Dosiseskalation von Chemotherapie, zu untersuchen und experimentelle Verfahren zur Überwindung von Chemoresistenz, z. B, durch Chemosensitivierung, zu entwickeln (1). Verwendet man genetisch modifizierte, chemoresistente Knochenmarkzellen im Sinne von Gentherapie und transplantiert diese, entstehen Transplantatempfänger, deren Knochenmark selektiv gegen die myelosuppressive Wirkung von Chemotherapie geschützt ist (2). Dies wird die Testung von Selektions- und Transplantationsprotokollen in der Gentherapie am Menschen wesentlich beschleunigen. Ferner kann natürlicherweise hochgiftiges Protein-Toxin, z. B. aus Pseudomonas Exotoxin, genetisch kloniert und in geeigneter Weise gezähmt werden, um therapeutisch nutzbar zu werden. Durch heterofunktionelle Kreuzkonjugation an einen monoklonalen Antikörper gegen das Gen- 

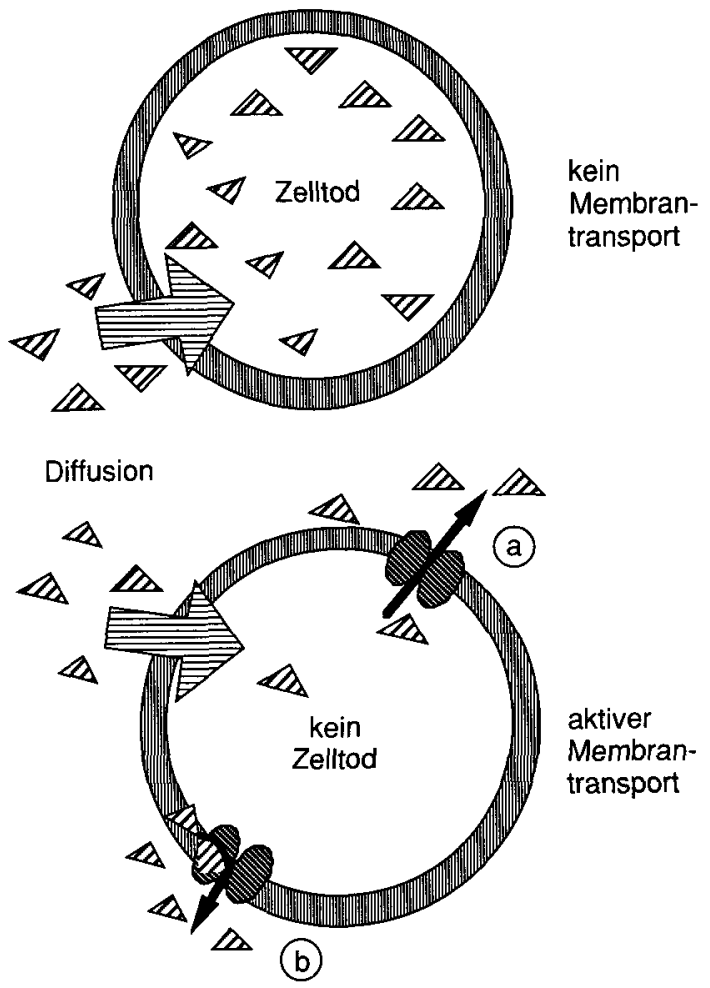

Abb. 6 Modell der Wirkungsweise des MDR1-Genproduktes, P-Glykoprotein zytotoxische Substanzen wie Chemotherapeutika gelangen natürlicherweise durch Diffusion in die Zelle. Dies führt in Zellen ohne P-Glykoprotein zum Zelltod, da hier kein auswärts gerichteter Membrantransport stattfindet. In Zellen, die durch P-Glykoprotein geschützt sind, wird durch aktiven Membrantransport aus a) dem Zytosol und b) bereits aus der Membran kein ausreichender Wirkspiegel erreicht, so daß die Zelle überlebt.

Nierenzellkarzinom MDR 1 Probe Actin

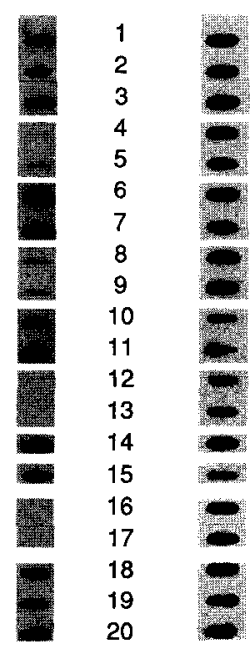

Zellinien

$\mathrm{KB}-$
Abb. 7 Nachweis des MDR1-Genes im Nierenzellkarzinom. Slot blot- Analyse unter Verwendung der molekularen Sonde MOR5A (2). Tumoren Nr. 1, 2, 3, 6, 7, 10, 11, 14, $15,18,19,20$ wiesen einen hohen, Tumoren Nr. 4, 5, 8, 9 einen niedrigeren MDR1 mRNAGehalt auf. Nr. 12, 13, 16, 17 sind negativ für MDR1-Transkripte. Die Expressionshöhe entspricht der Referenzzellinie KB-8-5. produkt des menschlichen MDR1 Genes entsteht ein Medikament, das immunologisch zielgerichtet chemoresistente Tumorzellen vernichtet, während es Zellen ohne entsprechende Antigenexpression verschont.

\section{Literatur}

${ }^{1}$ Mickisch, G. H., G. T. Merlino, H. Galski, M. M. Gottesman, I. Pastan: Transgenic mice that express the human multidrug resistance gene in bone marrow enable a rapid identification of agents that reverse drug resistance. Proc. Natl. Acad. Sci. USA, 88 (1991) $547-551$

2 Mickisch, G. H., I. Aksentijevich, P. V. Schoenlein, L. J. Goldstein, H. Galski, C. Stahle, D. H. Sachs, I. Pastan, M. M. Gottesman: Transplantation of bone marrow cells from tansgenic mice expressing the human MDR1 gene results in longterm protection against the myelosuppressive effect of chemotherapy in mice. Blood 79 (1992) 1087-1093

S. C. Müller et al. berichteten über die Verwendung von freier Mundschleimhaut zur Harnröhrenrekonstruktion.

Bei insgesamt 8 Patienten wurde autologe Mundschleimhaut als freies Transplantat zur Harnröhrenrekonstruktion verwendet. Indikationen waren:

„Hypospadie-Krüppel“ ( $\mathrm{n}=4)$, Syndrom der kurzen Urethra $(n=2)$, kontinente Epispadie $(n=1)$, Genitalrekonstruktion bei Blasenekstrophie $(\mathrm{n}=1)$. Wir benutzten die Mundschleimhaut aus folgenden Gründen:

Sie ist leicht erreichbar (Abb. 8), nicht haartragend und hat einen der penilen Harnröhre ähnlichen histologischen Aufbau.

Die intraorale Entnahmestelle zeigt kosmetisch hervorragende Resultate. Tierversuche am Hund verliefen zu unserer vollsten Zufriedenheit.

Bis auf 1 Patienten konnte die Harnröhrenrekonstruktion im Sinne eines einzeitigen Verfahrens durchgeführt werden. Das freie Mundschleimhauttransplantat wurde entweder als Rohr $(n=6)$ oder Patch $(n=2)$ eingesetzt. In 2 Fällen kam es zum kompletten bzw. partiellen Verlust des Transplantats mit entsprechender infravesikaler Obstruktion. 3 kleine Fisteln konnten problemlos sekundär versorgt werden. 3 Patienten galten primär als geheilt.

Trotz insgesamt enttäuschender Ergebnisse (Fisteln, Transplantatnekrose) erweitert dieses Verfahren die Möglichkeiten der Harnröhrenrekonstruktion vor allem dann, wenn Haut kaum mehr zur Verfügung steht. Eine verfeinerte Entnahmetechnik (Mukotom!), die ein dünnes Transplantat liefert, dürfte die Ergebnisse verbessern.

\section{Literatur}

R. A. Bürger, S. C. Müller, H. El-Damanhoury, A. Tschakaloff, $H$. Riedmiller, R. Hohenfellner: The Buccal Mucosal Graft for Urethral Reconstruction: A preliminary Report. J. Urol. 147 (1992) 662 

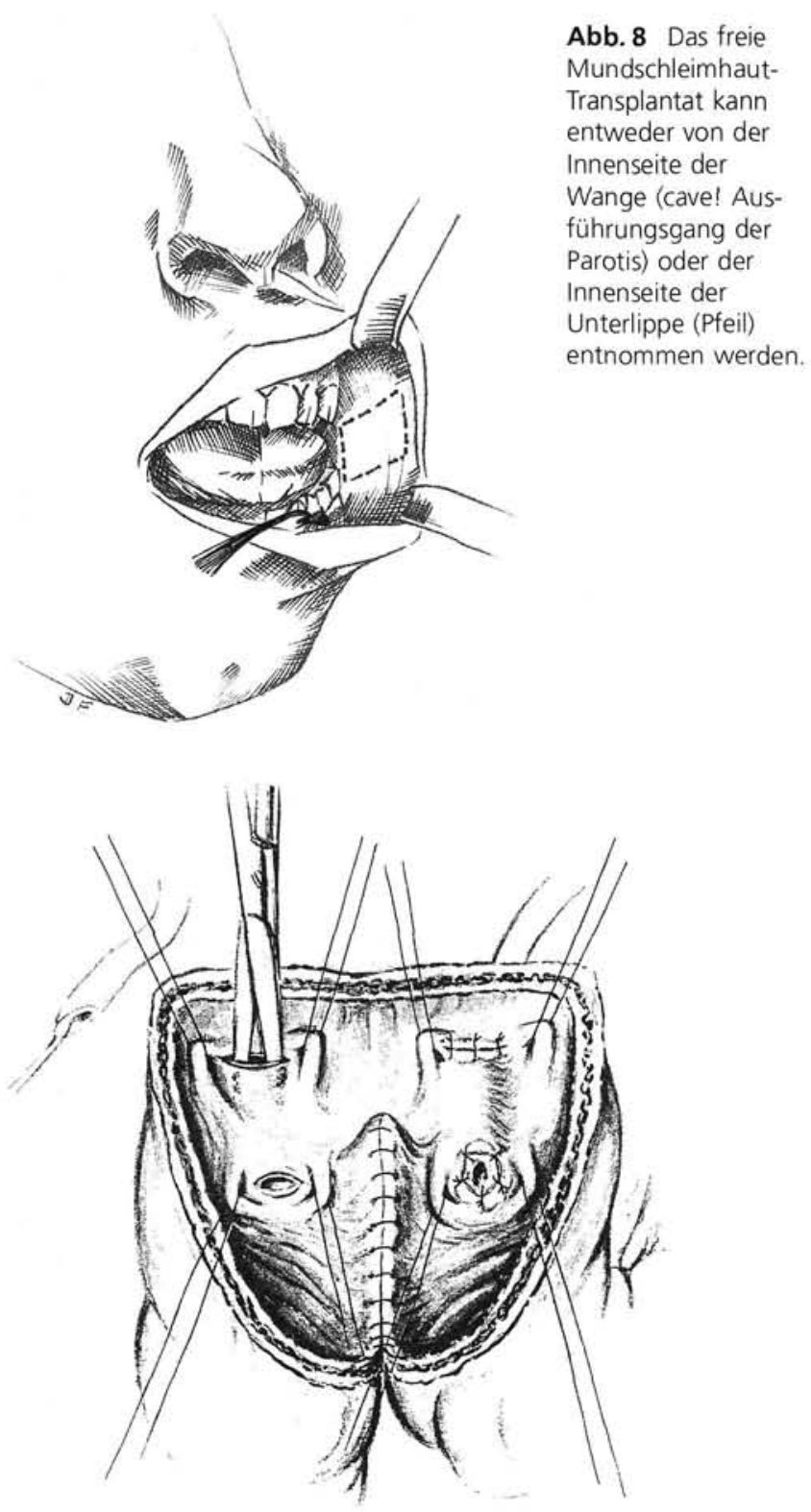

Abb. 9 Herstellung eines Sigma-Rektum-Pouch:

Die Naht der Hinterwand ist abgeschlossen. Die Harnleiter werden durch Knopflochinzisionen in das Pouchlumen eingezogen und dann durch submuköse Tunnel refluxgeschützt eingenäht.

Die kontinente Harnableitung ist heute allgemein als Methode der 1. Wahl akzeptiert. In diesem Zusammenhang war der abschließende Vortrag von R. Hohenfellner (Mainz) interessant, der eine modifizierte Methode der Harn-Umleitung, den Mainz-Pouch II, vorstellte.

Die Harnleiterdarmimplantation ist das älteste Verfahren der kontinenten Harnableitung. Das Risiko rezidivierender Pyelonephritiden und metabolischer Entgleisungen konnte durch operationstechnische Verbesserung der antirefluxiven Harnleiterimplantation und Entwicklung potenter Pharmaka (Antibiotika, alkalysierende Substanzen) vermindert werden. Obgleich dieses Verfahren in den vierziger und fünfziger Jahren fast völlig verlassen wurde, gewinnt es heutzutage aufgrund seiner einfachen Durchführbarkeit und geringen Komplikationsrate wieder zunehmend an Bedeutung.

Bislang stellten symptomatische und asymptomatische Harntransportstörungen nach HDI jedoch noch ein Problem dar. Eine wesentliche Ursache ist die zu hohe Harnleiterimplantation in eine "falsche Schlinge“. Durch die enorme Mobilität des Sigmas und dadurch bedingter Lageveränderungen kommt es zu Abknickungen des Harnleiters.

Das nachfolgend beschriebene Verfahren wurde primär als Revisionsoperation nach fehlgeschlagener HDI entwickelt. Jedoch zeigte sich, daß dieses Operationsverfahren gegenüber der klassischen HDI einige wesentliche Vorteile bietet und somit diese ersetzen kann.

Beginnend am rektosigmoidalen Übergang wird der Darm entlang der Taenia libera über je $10-12 \mathrm{~cm}$ nach distal und proximal längs eröffnet. Der eröffnete Darm läßt sich in Form eines umgekehrten V legen. Durch Seit-zu-SeitAnastomose der Hinterwand (mediale Ränder des V) wird eine Pouchplatte gebildet. Der proximale Endpunkt der Anastomose läßt sich mittels zweier Bassini-Nähte am Periost des Promontoriums fixieren. Zuvor erfolgt die antirefluxive Harnleiterimplantation unter Verwendung der Goodwin-Hohenfellner Technik (Abb. 9). Der Einsatz der „open-end“-Technik erlaubt auch die Implantation dilatierter Harnleiter. Nach Verschluß der Pouchvorderwand und der Mesenterialinzision sollte, wenn möglich, der Anastomosenbereich durch Netz gedeckt werden.

Bislang erhielten 37 Patienten, einschließlich 6 Kinder an unserer Klinik einen Mainz-Pouch II. Es wurden bei einer Beobachtungszeit bis zu 18 Monaten keine wesentlichen Früh- oder Spätkomplikationen gefunden.

Urodynamische Messungen zeigen, daß durch diese Operationstechnik ein Niederdruckreservoir geschaffen wird, wobei hohe Druckspitzen durch Darmkontraktionen eliminiert werden. Dies schützt einerseits den oberen Harntrakt und verbessert andererseits die Kontinenzrate. Der gerade Harnleiterverlauf und die stabile Fixierung des Reservoirs im Bereich des Promontoriums stellen darüber hinaus wesentliche Vorteile der Mainz-Pouch II dar.

\section{Literatur}

${ }^{1}$ Fisch, M., R. Hohenfellner: Sigma-Rektum Pouch: Eine Modifikation der Harnleiterdarmimplantation. Akt. Urol. 22 (1991) $\mathrm{I}-\mathrm{X}$

${ }^{2}$ Fisch, M., R. Wammack, S. C. Müller, R. Hohenfellner: The Sigma-rectum Pouch (MAINZ Pouch II). J. Urol. (1992) in press

Aus der Beurteilung des Organisators war dieses 9. Treffen der E. C. Alken-Preisträger ein voller Erfolg. In einer familiären und relaxierten Umgebung trafen sich Freunde und Experten der verschiedenen Teilgebiete der Urologie, die sich alle auf das folgende Teffen 1992 in Heidelberg freuen.

\section{Udo Jonas}

Urologische Klinik

Medizinische Hochschule Hannover

Konstanty-Gutschow-Str. 8

3000 Hannover 61 